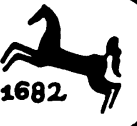

Sammlung Metzler Band 234 


\section{Deutsche \\ und europäische Barockliteratur}

J.B. Metzlersche Verlagsbuchhandlung Stuttgart 


\section{CIP-Kurztitelaufnahme der Deutschen Bibliothek}

Hoffmeister, Gerhart:

Deutsche und europäische Barockliteratur / Gerhart Hoffmeister.

- Stuttgart : Metzler, 1987.

(Sammlung Metzler ; Bd. 234)

ISBN 978-3-476-10234-8

NE: GT

ISBN 978-3-476-10234-8

ISBN 978-3-476-03937-8 (eBook)

DOI 10.1007/978-3-476-03937-8

ISSN 05583667

\section{SM 234}

Dieses Werk einschließlich aller seiner Teile ist urheberrechtlich geschützt. Jede Verwertung außerhalb der engen Grenzen des Urheberrechtsgesetzes ist ohne Zustimmung des Verlages unzulässig und strafbar. Das gilt insbesondere für Vervielfältigungen, Übersetzungen, Mikroverfilmungen und die Einspeicherung und Verarbeitung in elektronischen Systemen.

(c) Springer-Verlag GmbH Deutschland 1987

Ursprünglich erschienen bei J. B. Metzlersche Verlagsbuchhandlung und Carl Ernst Poeschel Verlag GmbH in Stuttgart 1987 
Vorbemerkung $\ldots \ldots \ldots \ldots \ldots \ldots \ldots \ldots$ VII

Abkürzungen $\ldots \ldots \ldots \ldots \ldots \ldots$ IX

Öfter genannte Sekundärliteratur $\ldots \ldots \ldots \ldots$ XI

I. Zur Begriffsgeschichte von »Barock $« \ldots \ldots \quad 1$

II. Nationale Spielarten des Barock . . . . . . . . 9

1. Italienischer Barock . . . . . . . . . . . 9 9

2. Spanischer Barock . . . . . . . . . . . 15

3. Französischer Barock . . . . . . . . . . . 23

4. Englischer Barock . . . . . . . . . . . . 32

5. Holländischer Barock . . . . . . . . . . . . 40

6. Skandinavischer Barock $\ldots \ldots \ldots \ldots \ldots .445$

7. Slawischer Barock . . . . . . . . . . . 48

8. Deutscher Barock . . . . . . . . . . . 56

III. Barocke Wechselbeziehungen $\ldots \ldots \ldots \ldots 68$

1. Italien und Deutschland . . . . . . . . . . 68

2. Spanien und Deutschland . . . . . . . . . 77

3. Frankreich und Deutschland . . . . . . . . 88

4. England und Deutschland . . . . . . . . . 93

5. Holland und Deutschland . . . . . . . . . . 97

6. Deutschland und Skandinavien . . . . . . . . . 105

7. Deutscher und Slawischer Barock . . . . . . 107

IV. Europäische Perspektiven $\ldots . \ldots \ldots \ldots .112$

1. Gibt es eine europäische Barockliteratur? . . . 112

2. Soziale Grundlagen der Barockliteratur . . . . . 114

3. Die lateinische Dichtungstradition . . . . . . . 127

4. Rhetorik und Emblematik . . . . . . . . . . . 134

5. Jesuitendichtung . . . . . . . . . . 140

6. Marinismus . . . . . . . . . . . . . . 147 
7. Gattungen ....................... 156

8. Themen, Motive, Gestalten . . . . . . . . 172

V. Zur Wirkungsgeschichte der Barockliteratur in Deutschland . . . . . . . 186

Personenregister . . . . . . . . . . . . . . 199 


\section{Vorbemerkung}

"Man muß sich in unseren neueren

Zeiten auf einen europäischen

Standpunkt erheben "

(Mme de Staël, »De l’Allemagne«, 1813)

Wie der Parallelband über die literarischen Wechselbeziehungen zwischen der "Deutschen und europäischen Romantik " (1978) entspringt auch diese Arbeit dem Wunsch des Verfassers, einen gewissen Überblick über den derzeitigen Stand der Barockforschung zu gewinnen, soweit sie von einem komparatistischen Ansatz ausgehend die deutsche Barockliteratur als Teil der Weltliteratur versteht. Zweifellos war sie Glied der internationalen Gelehrtenrepublik, die aus der Kontinuität der lateinischen und christlichen Tradition lebte und wirkte. Vor allem in der ersten Hälfte des Barockjahrhunderts wurden die Fundamente der neueren deutschen Literatur gelegt, und zwar durch Imitation und Wetteifer mit der gesamteuropäischen Dichtungstradition, deren Zusammenhang sich, über rein positivistische Quellenforschung hinaus, durch vergleichende Studien erschließen läßt: »denn wo findet man einen solchen Reichtum an internationalen Rezeptionssträngen, eine solche Fülle von Traditionsverflechtungen über die Grenzen hinweg und eine solche Freizügigkeit der Gattungs- und Stilformen wie im Barock? " (Lunding 1975, S. 193). Dank dieser Einsicht hat sich seit den sechziger Jahren eine zunehmende Internationalisierung der Barockforschung durchgesetzt, die im Zeichen von Rezeptions- und Produktionsstudien überzeugende Gegenbeispiele für die oft beklagten nationalen Scheuklappen in der Literaturwissenschaft geliefert hat. Dabei ist sowohl an den »Internationalen Arbeitskreis für deutsche Barockliteratur « in Wolfenbüttel (ab 1972) als auch die amerikanische "Society for German Renaissance and Baroque Literature" (MLA, New York) zu denken; hinzu kommen die Arbeiten der G. Weydt-Schule in Münster (siehe Bibliographie) und L. W. Forsters und M. Birchers Ausgaben und Studien zur europäischen Barockliteratur.

$\mathrm{Zu}$ einem Thema derartiger Spannweite sind die Grundlagen seit Jahrzehnten gelegt worden: durch die Barockstudien des Verfassers unter Richard Alewyn und Günther Weydt in Bonn 
(1957-1963) und Blake L. Spahr in Berkeley (1963-64), durch die eigenen komparatistischen Arbeiten, Editionen und Sammelbände vor allem zu den spanisch-deutschen und italienischdeutschen Beziehungen, durch die in der Sammlung Metzler bereits erschienenen "Bausteine « zur Barockliteratur (V. Meid, Der dt. Barockroman, 1974; R. Alexander, Das dt. Barockdrama, 1984) und durch jahrzehntelange Zusammenarbeit und Korrespondenz mit führenden Barockgelehrten in Deutschland und Österreich, Holland und den USA.

Für stete Förderung gebührt ihnen allen der Dank des Verfassers. Die Verantwortung für die hier vorgelegte Übersicht trägt er allein. Und sie ist nicht gering zu schätzen, da es keine leichte Aufgabe war, die jüngste Entwicklung der dt. Barockforschung von Santa Barbara aus zu verfolgen. Andererseits ist diesem Blickwinkel sowohl die Darstellung der dt. Literatur des 17. Jh.s aus dem europäischen Kontext als auch die Beachtung eines möglichst lesbaren Stils bei aller Komplexität des Gegenstandes zu verdanken. Diese Arbeit wurde noch ohne Computer-Hilfe fertiggestellt.

G. H. 


\section{Abkürzungen}

Bibl.

$=$ Bibliographie

Cent.

$=$ Century

CollG.

= Colloqia Germanica

CRCL

$=$ Canadian Review of Comparative Literature

D., i. D. = Druck, im Druck

Dok. $\quad=$ Dokument,-e

Dt. Philol.i.A. = Deutsche Philologie im Aufriß, hrsg. W. Stammler. 3 Bde. $1951 \mathrm{ff},{ }^{2} 1955 \mathrm{ff}$.

DU = Der Deutschunterricht

DVJ = Deutsche Vierteljahrsschrift für Literaturwissenschaft und Geistesgeschichte

EG = Études Germaniques

Fs.

$=$ Festschrift

GLL $=$ German Life and Letters

GQ

GRM

$=$ German Quarterly

$=$ Germanisch-Romanische Monatsschrift

HA

= Hamburger Ausgabe (Goethe, hrsg. E. Trunz)

Hdb. $\quad=$ Handbuch

Hrsg(g)., hrsg. = Herausgeber, herausgegeben

IADBL = Internationaler Arbeitskreis für deutsche Barockliteratur (Wolfenbüttel)

IASL = Internationales Archiv für Sozialgeschichte der Literatur (Wien)

IGK = Internationaler Germanistenkongreß

Jb. $\quad=$ Jahrbuch

JDSG = Jahrbuch der deutschen Schillergesellschaft

K.-W. $\quad$ = D. Kimpel, C. Wiedemann, Hrsgg.

MLN = Modern Language Notes

MLQ = Modern Language Quarterly

Ms. $\quad=$ Manuskript

NHdb. d. = Neues Handbuch der Literaturwissen-

Litwiss. schaft, hrsg. August Buck, 1972. 


$\begin{array}{ll}\text { Propyläen } & =\text { Propyläengeschichte der Literatur } \\ \text { Repr. } & =\text { Reprint } \\ \text { RFH } & =\text { Revista de Filología Hispánica } \\ \text { RL } & =\text { Reallexikon der deutschen Literatur- } \\ & \text { geschichte }{ }^{1} 1925 \mathrm{f.} .{ }^{2} 1955 \mathrm{f} . \\ \text { Slg. } & =\text { Sammlung } \\ \text { SN } & =\text { Studia Neophilologica } \\ \text { S. W. } & =\text { Sämtliche Werke } \\ \text { Übers(s). } & =\text { Übersetzer } \\ \text { Unters(s). } & =\text { Untersuchung,-en } \\ \text { WB } & =\text { Weimarer Beiträge } \\ \text { WBN } & =\text { Wolfenbütteler Barock-Nachrichten }\end{array}$




\section{Öfter genannte Sekundärliteratur}

Aiken $1982=$ Aiken, J.: German Baroque Drama. Boston 1982.

Alewyn $1926=$ Alewyn, R.: Vorbarocker Klassizismus u. griech. Tragödie.

Alewyn-Sälzle 1959 = Das große Welttheater. Die Epochen der höfischen Feste in Dokument und Deutung.

Alexander $1984=$ Alexander, R. J.: Das dt. Barockdrama. Slg. Metzler 209.

Angyal 1975 = Angyal, A.: Das Problem des slawischen Barocks (195657), in: W. Barner: Der literar. Barockbegriff 1975, S. 329-59.

Asmuth $1971=$ Asmuth, B.: Lohenstein und Tacitus.

Barner BR 1970 = Barockrethorik: Untersuchungen zu ihren geschichtlichen Grundlagen.

Barner 1975 = Der literar. Barockbegriff. Wege der Forschung 358 .

Barock-Dokumente 1965 = Alewyn, R., Hrsg.: Deutsche Barockforschung, Dokumentation einer Epoche. 1965, 21966.

Benjamin 1928 = Benjamin, W.: Ursprung des deutschen Trauerspiels. $1928,{ }^{2} 1963,{ }^{3} 1969$.

Buck $1980=$ Buck, A.: Forschungen zur romanischen Barockliteratur.

Catholy $1968=$ Das deutsche Lustspiel. I: Vom Mittelalter bis zum Ende der Barockzeit.

Conrady 1962 = Conrady, K. O.: Lateinische Dichtungstradition und deutsche Lyrik des 17. Jahrhunderts.

Curtius $1948=$ Curtius, E. R.: Europäische Literatur und lateinisches Mittelalter. 1948, ${ }^{6} 1967$.

Deutsche Barockliteratur 1977 = Bircher, M. und E. Mannack, Hrsgg.: Dt. Barockliteratur und europäische Kultur. Dokumente des IADBL 3.

Dichter $1984=$ Steinhagen, H. und B. von Wiese, Hrsgg.: Deutsche Dichter des 17. Jahrhunderts. Ihr Leben und Werk.

Elwert 1972 = Elwert, W. Th.: Die Lyrik der Renaissance und des Barocks in den romanischen Ländern, in: NHdb d. Litwiss. IX, S. 82127.

Europäische Hofkultur 1981 = Buck, A. et al., Hrsgg.: Europ. Hofkultur im 16. u. 17. Jh. 3 Bde.

Europäische Rezeption 1983 = Forster, L. W., Hrsg.: Studien zur europ. Rezeption dt. Barockliteratur. Wolfenbütteler Arbeiten zur Barockforschung 11.

Europäische Tradition 1973 = Hoffmeister, G., Hrsg.: Europ. Trad. und deutscher Literaturbarock. 
Festschrift Weydt $1972=$ Rasch, W. et al., Hrsgg.: Rezeption und Produktion zwischen 1570 und 1730. Bern.

Formkräfte $1963=$ Steffen, H. Hrsg.: Formkräfte der deutschen Dichtung.

Forster $1977=$ Deutsche und europäische Barockliteratur, in: Daphnis 6, 1977, S. 31-56.

Friederich 1954 = Friederich, W. P.: The Baroque, in: Outline of Comparative Literature, S. 99-153. Chapel Hill.

Friedrich 1954 = Friedrich, H.: Epochen der italienischen Lyrik.

Gaede 1971 = Gaede, F.: Humanismus, Barock, Aufklärung (= Geschichte der deutschen Literatur I, 2) Bern.

German Baroque Literature 1983 = Hoffmeister, G. Hrsg.: German Baroque Literature; The European Perspective. New York.

Geulen 1975 = Geulen, H.: Erzählkunst der frühen Neuzeit.

Hankamer 1935 = Hankamer, P.: Deutsche Gegenreformation und deutscher Barock. ${ }^{3} 1964$.

Hinck $1965=$ Hinck, W.: Das deutsche Lustspiel des 17. und 18. Jahrhunderts und die italienische Komödie.

Hocke 1959 = Hocke, G. R.: Manierismus in der Literatur. Sprachalchemie und esoterische Kombinationskunst (rde.).

Hof, Staat 1982= Blüm, E. et. al., Hrsgg.: Hof, Staat und Gesellschaft in der Literatur des 17. Jahrhunderts. Amsterdam 1982.

Hoffmeister 1973 = Barocker Petrarkismus. Wandlungen und Möglichkeiten der Liebessprache in der Lyrik des 17. Jahrhunderts, in: Europäische Tradition 1973, S. 37-55.

Hoffmeister $1976=$ Spanien und Deutschland. Geschichte und Dokumentation der literarischen Beziehungen (Span. Ausgabe: España y Alemania. Madrid 1980).

Hoffmeister 1986 = Prolegomena zu einer Literaturgeschichte des europäischen Barock, in: Festschrift Remak, S. 125-37.

Iser $1960=$ Iser, W.: Manieristische Metaphorik in der engl. Dichtung, in: GRM 41, 1960, S. 266 f.

Jantz 1962 = Jantz, H.: German Baroque Literature, in: MLN 77, 1962, S. 337-67.

Jantz 1973 = Die Erforschung des Barock. Beobachtungen und Erfahrungen, in: IADBL 1, S. 3-20.

Kindermann 1959 = Kindermann, H.: Theatergeschichte Europas. III: Das Theater der Barockzeit. Salzburg.

Kühlmann 1982 = Kühlmann, W.: Gelehrtenrepublik und Fürstenstaat.

Kunstformen 1956 = Stamm, R., Hrsg.: Die Kunstformen des Barockzeitalters. Bern.

Literary Culture $1988=$ Parente, J. und R. Schade, Hrsg.: Literary Culture in the Holy Roman Empire. Chapel Hill 1988 (i. D.).

Lunding 1975 = Lunding, E.: Komparatistische Barockforschung mit bes. Berücksichtigung Dänemarks, in: Teilnahme und Spiegelung. Fs. H. Rüdiger, S. 193-216.

Meid $1974=$ =Meid, V.: Der deutsche Barockroman. Slg. Metzler 128. 
Mirollo 1963 = Mirollo, J. V.: The Poet of the Marvellous. Giambattista Marino. New York - London.

Montano $1967=$ Montano, R.: Metaphysical and Verbal arguzia and the Essence of the Baroque, in: CollG, 1, 1967, S. 49-65.

Müller 1929= Müller, G.: Höfische Kultur der Barockzeit, in: H. Naumann und G. M.: Höfische Kultur.

NHdb. d. Litwiss. 1972 = Buck, A., Hrsg.: Renaissance und Barock. Bd. IX und X.

Petrarkistische Lyrik $1973=$ Hoffmeister, G. Slg. Metzler 119.

Praz 1966 = Praz, M.: The Flaming Heart (1958). Gloucester, Mass. 1966.

Propyläengeschichte $1984=$ Renaissance und Barock 1400-1700. Propyläengeschichte der Literatur III.

Rötzer 1972 = Rötzer, H. G.: Der Roman des Barock 1600-1700. Winkler Kommentar.

Rousset $1954=$ Rousset, J.: La Littérature de l'âge baroque en France: Circe et la Paon. Paris 1954, ${ }^{2} 1960$.

Schäferdichtung $1977=$ Voßkamp, W., Hrsg.: Schäferdichtung. IADBL 4.

Schöffler $1956=$ Schöffler, H.: Dt. Geistesleben zwischen Reformation und Aufklärung. Von Opitz zu Chr. Wolff. 1940, ${ }^{2} 1956$.

Scholte 1958 = Scholte, J. H.: Barockliteratur in: RL I ${ }^{2} 1958$, S. 135-39.

Skrine $1978=$ Skrine, P. N.: The Baroque. Literature and Culture in 17 th Century Europe. London.

Sozialgeschichte 1985 = Steinhagen, H., Hrsg.: Zwischen Gegenreformation und Frühaufklärung: Späthumanismus, Barock 1572-1740 (= Dt. Lit. Eine Sozialgesch. Bd. III).

Sprachgesellschaften $1978=$ Bircher, M. und Van Ingen, Hrsgg.: Sprachgesellschaften, Sozietäten, Dichtergruppen.

Stadt - Schule 1976 = Schöne, A., Hrsg.: Stadt, Schule, Universität, Buchwesen in der dt. Literatur im 17. Jahrhundert.

Strich 1916 = Strich, F.: Der lyrische Stil des 17. Jahrhunderts (1916), in: Barock-Dokumente 1965, S. 229-59, oder Barner 1975, S. 32-71.

Strich 1947 = Der europäische Barock, in: F. S.: Der Dichter und die Zeit. Bern 1947, S. 71-131.

Strich 1956 = Die Übertragung des Barockbegriffs von der bildenden Kunst auf die Dichtung (1956), in: Barner 1975, S. 307-28.

Szyrocki 1968 = Szyrocki, M.: Die deutsche Literatur des Barock. Eine Einführung. 1968, ${ }^{2} 1979$.

Theatrum Europäum 1982 = Brinkmann, R. et. al. Hrsgg.: Theatrum Europäum. Festschrift E. M. Szarota.

Villwock 1984 = Villwock, J.: Rhetorik und Poetik: Theoretische Grundlagen der Literatur, in: Propyläengesch. III, S. 98-120.

Warnke $1972=$ Warnke, F. J.: Versions of Baroque. European Literature in the 17th Century. New Haven-London. 
Wellek $1965=$ Wellek, R. Der Barockbegriff in der Literaturwissenschaft (engl. 1946), in: Grundbegriffe der Litwiss. 1965, S. 57-94.

Weydt $1968=$ Weydt, G.: Nachahmung und Schöpfung. Studien um Grimmelshausen.

Windfuhr BB $1966=$ Windfubr, M.: Die barocke Bildlichkeit und ihre Kritiker. Stilhaltungen in der dt. Lit. des 17. und 18. Jh.s.

Zielske $1984=$ Zielske, H.: Drama und Theater in England, den Niederlanden und Deutschland, in: Propyläengeschichte III, S. 131-74. 